\title{
Chronic Kidney Disease in Japan from Early Predictions to Current Facts
}

\author{
Kunitoshi Iseki \\ Dialysis Unit, University Hospital of The Ryukyus, Okinawa, Japan
}

\begin{abstract}
Key Words
Asian Forum of CKD Initiative $\cdot$ Chronic kidney disease $\cdot$ CKD, incidence and prevalence $\cdot$ CKD, screening $\cdot$ End-stage renal disease $\cdot$ Frontier of Renal Outcome Modifications in Japan · Japanese Society for Dialysis Therapy
\end{abstract}

\begin{abstract}
Chronic kidney disease (CKD) is considered a risk factor for both end-stage renal disease (ESRD) and cardiovascular disease. Early detection and treatment of CKD is likely to be the key factor to prevent them. According to the Japanese Society for Dialysis Therapy, the prevalence of treated ESRD has increased to $>2,000$ per million population. More than $40 \%$ of ESRD incidents are due to diabetes mellitus. The Japanese Society of Nephrology estimates the prevalence of a low GFR $\left(<60 \mathrm{ml} / \mathrm{min} / 1.73 \mathrm{~m}^{2}\right)$ to be around $10 \%$ of the communitybased screening participants. However, such a high CKD prevalence requires detailed and critical confirmatory studies and analyses, many of which are currently underway in Japan.

Copyright $\odot 2008$ S. Karger AG, Base
\end{abstract}

\section{Introduction}

The presumed advantage of early detection of chronic kidney disease (CKD) is to decrease end-stage renal disease (ESRD) and other related medical complications through effective treatment strategies [1]. Low GFR is now widely accepted as a risk factor for cardiovascular

\section{KARGER}

Fax +4161306 1234 E-Mail karger@karger.ch www.karger.com

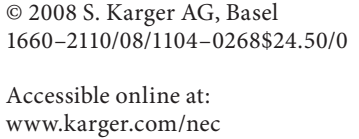

disease (CVD) and mortality [2, 3], although proteinuria has been known as a risk factor for CVD and mortality. The definition of CKD based on eGFR is simple and easy to understand, and helpful for practitioners to detect unrecognized CKD patients. The CKD population is large. Therefore multidisciplinary collaborations among physicians, healthcare professionals, general physicians, and the government are necessary to address issues related to $\mathrm{CKD}$, its progression and complications.

According to the Japanese Society for Dialysis Therapy, the prevalence of patients with treated ESRD on chronic dialysis therapy has been $>2,000$ per million population since 2005 [4] and it increased to 2,153 per million population at the end of 2007. The incidence of treated ESRD is also increasing despite several strategies, including universal screening and the use of presumably effective drugs [5]. For instance, in Okinawa, where over the years a number of CKD screening programs and strategies have been conducted, there has been a steep rise in ESRD prevalence (fig. 1). The rising ESRD prevalence could be explained by multiple factors such as better survival on dialysis therapy in Japan [6], an insurance system to cover dialysis therapy thus allowing bigger access, and 'truly' increased incidence and prevalence of CKD leading to ESRD. A growing elderly and diabetic population may also contribute to this trend as shown elsewhere in the world.

Against these backgrounds, the Japanese Society of Nephrology (JSN) has distributed more than 140,000 copies of the 'CKD Practice Guide' published in September 2007 (www.jsn.or.jp). This booklet, in total 116 pages, was planned to spread the concept of CKD amongst gen-

Dr. Kunitoshi Isek

Dialysis Unit, University Hospital of The Ryukyus

207 Uehara, Nishihara, Okinawa 903-0215 (Japan)

Tel. +8198895 1341, Fax +81988951416

E-Mail chihokun@med.u-ryukyu.ac.jp 
eral practitioners to improve $\mathrm{CKD}$ care and decrease the development of ESRD and CVD amongst this high-risk population. Criteria for referral to nephrologists are: (1) proteinuria $=>2+$ by dipstick or $>0.5 \mathrm{~g} / \mathrm{g}$ creatinine, $(2)$ combined proteinuria and hematuria (both, $1+$ or over by dipstick), and (3) eGFR $<50 \mathrm{ml} / \mathrm{min} / 1.73 \mathrm{~m}^{2}$. The JSN recommends testing urine for proteinuria and serum creatinine among those who are on treatment for hypertension, diabetes mellitus (DM), CVD, and among the elderly population.

\section{Screening of CKD}

Japan has a long history of universal screening [7]. In the early 1970s, mandatory kidney disease screening was started including urinalysis of all workers and school-age children. In 1983, nationwide urinalysis screening for adults aged 40 years and over, and in 1992 measurement of serum creatinine, was mandated in the communitybased health examination program. Gathering such nationwide screening data, the JSN estimated the prevalence of stage 3 CKD was $10.4 \%$ in more than half a million participants. Among $7.6 \%$ of the subjects the GFR were in the range of $50-59 \mathrm{ml} / \mathrm{min} / 1.73 \mathrm{~m}^{2}$. The high percentage of this borderline group with known tendency of fluctuations in and out of the CKD range (eGFR $<60 \mathrm{ml} /$ $\mathrm{min}$ ) warrants some caution when interpreting the overall prevalence of CKD in the general population. This group $(7.6 \%)$ may also include a large number of elderly individuals with age-related decreased GFR and doubtful significance. However, in view of doubts over the applicability of estimated GFR based on the conventional MDRD formula to the Japanese population, the JSN came up with an alternative Japanese GFR estimation equation that was validated by GFR measured with inulin clearance: $\mathrm{eGFR}=194 \times \mathrm{Scr}^{-1.094} \times \mathrm{Age}^{-0.287}$, multiplied by 0.739 , if female [S. Matsuo et al., unpubl. observations]. We also validated the MDRD Study equation for Japanese: $\mathrm{eGFR}=0.741 \times 175 \times \mathrm{Scr}^{-1.154} \times \mathrm{Age}^{-0.203}$, multiplied by 0.742 , if female [7]. Both formulae use serum creatinine measured by the enzymatic method.

\section{Incidence and Prevalence of CKD}

The risk of developing ESRD among those without any known risk factors is very low, namely 1 per million per year [5]. The optimal time to offer therapy to asymptomatic subjects at low risk is not clear. The cost-benefit

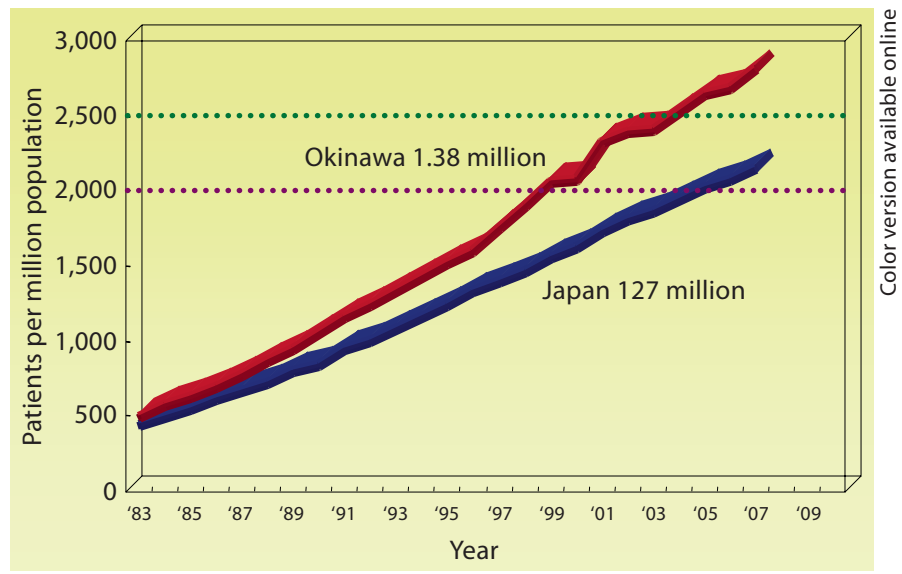

Fig. 1. Trends in prevalence of ESRD on chronic dialysis. Data were taken from the Japanese Society for Dialysis Therapy.

analysis concerning the frequency and extent of screening has not yet been performed in Japan.

The age-related decline in GFR is not as high as expected, as it was $0.36 \mathrm{ml} / \mathrm{min} / 1.73 \mathrm{~m}^{2} /$ year in the general Japanese population [8]. Rate of decline in GFR was faster when the baseline GFR was $<50 \mathrm{ml} / \mathrm{min} / 1.73 \mathrm{~m}^{2}$. However, it is unclear whether those with eGFR $<50 \mathrm{ml} /$ $\min / 1.73 \mathrm{~m}^{2}$ have an underlying nephropathy. Of note, decreased age-related GFR does not progress to CKD stage 3 unless associated with hypertension or DM supporting such an assumption.

The CKD prevalence seemed to be increasing in Japan according to the community-based study in Hisayama [9]: the age-adjusted prevalence of stage 3 and 4 CKD was $4.1 \%$ in $1974,4.8 \%$ in 1988 , and $8.7 \%$ in 2002 in men, and $7.3 \%$ in $1974,11.2 \%$ in 1988 , and $10.7 \%$ in 2002 in women. All those subjects screened were 40 years and over at screening and the age-adjusted prevalence increased significantly in both men and women compared to that of 1974. It is noteworthy that the prevalence of obesity, BMI $\geq 25 \mathrm{~kg} / \mathrm{m}^{2}$, increased in men ( $11 \%$ in $1974,24 \%$ in 1988 , and $29 \%$ in 2002). According to the recently published studies the significant impact of metabolic syndrome may at least in part explain the increased tendency of CKD incidence among screened subjects $[10,11]$. This association may be related to both genetic and environmental factors. Those with low birth weight, which is associated with lower nephron number, may be predisposed to insulin resistance and therefore may be at increased risk of proteinuria and CKD. Also, lifestyle-related factors that are often associated with obesity and metabolic syn- 
drome may have a role in the development and progression of CKD. Among the lifestyle-related factors, smoking is a known risk factor of CKD [12]. Regulations of smoking are becoming tighter in Japan. Currently, it is prohibited in public places. ID cards are required to buy tobacco from vending machines.

\section{Predictors of CKD and ESRD}

We have been studying the predictors of treated ESRD by using community-based screening programs and the ESRD registry in Okinawa, Japan [13, 14]. Using two registries, we identified screening participants who later entered a dialysis program. Important predictors of developing ESRD are dipstick-positive proteinuria and hypertension. Both predictors are easy to perform and therefore suitable screening tools, especially in developing countries. Dipstick proteinuria predicted cardiovascular mortality in a large cohort study, suggesting that simple tests may help in reliably predicting a hard outcome [15]. Low GFR per se, which is often observed in the elderly population, is not a strong predictor of developing ESRD unless associated with proteinuria [16]. The combination of low GFR and proteinuria in the elderly may identify those with underlying nephropathies compared to those with potentially benign age-related decreased GFR. In this regard, studies are necessary to clarify such an important issue as a large percentage of those with putative CKD may simply have age-related low GFR with low risk of progression of ESRD.

A number of prospective studies have been initiated in Japan to address many of the issues related to CKD.

\section{FROM-J: Frontier of Renal Outcome Modifications in} Japan

Due to the high prevalence and incidence of ESRD, the Japanese Government (Ministry of Health and Labor) assigned CKD as a national target disease for the strategic medical research in 2007. The Japan Kidney Foundation was asked to launch a CKD study aimed at analyzing the impact of treatment intensification on CKD management by using a cluster-random method. The priority of this project is to observe the referral rate of CKD patients from general physician to nephrologists. Therefore, the precise rate of CKD progression and event rate related to CKD stages may not be critically evaluated. Treatment strategies will be randomly assigned to each regional medical association. Each medical association has registered 10 general physicians (Kakarituke doctors). A total of 50 regional medical associations will participate, i.e. 500 general physicians. Each physician will have registered at least 5 CKD patients by the end of September 2008. In both treatment groups, CKD patients will be treated by a general physician based on the CKD practice guidelines issued by the JSN in 2007. The difference between the groups will focus on the intensity of treatment such as follow-up monitoring frequency, lifestyle modifications and dietary instruction. The primary outcomes are: (1) the dropout rate, (2) the referral rate to registered nephrologists, and (3) the progression rate of CKD to ESRD. The strengths of this project are that this is the first national project to fight $\mathrm{CKD}$ and alert co-medical staff such as nurses, dietitians, and other medical services to it. CKD practice guidelines will be disseminated throughout Japan by this study.

\section{CKD-JAC: Chronic Kidney Disease-Japan Cohort [17]}

The natural history and course of CKD has not been studied in a large cohort of patients. Risk factors of CKD progression with respect to the development of CVD are not known in Japan. The study will enroll 3,000 CKD patients, eGFR $10-59 \mathrm{ml} / \mathrm{min} / 1.73 \mathrm{~m}^{2}$, in 18 clinical centers around Japan. Each clinical center will enroll approximately 200 patients over 12 months. They will monitor the incidence of ESRD, CVD, and all-cause mortality for 4 years. The study will also examine the relationship between CKD and quality of life. The enrolment was started in September 2007. The strength of this study is that it is conducted nationwide and the number of patients is large. It is expected to provide clues to estimate the cost of treatment among the identified CKD patients for preventing ESRD. The limitations of the study are that CKD treatment may differ among facilities, therefore it may not provide evidence for the efficacy of CKD treatment strategies. Enrolled facilities are well equipped and prestigious, therefore they may not represent the current practice in Japan.

\section{AFCKDI: Asian Forum of CKD Initiative}

The Asian Forum of CKD Initiative (AFCKDI) was launched on 2007, Hamamatsu, Japan. This Forum was proposed by the JSN and made possible with the collaboration of ISN, COMGAN, APSN, and other Asian ne- 
Table 1. Possible causes of high prevalence of ESRD in Japan [data from 5]

Genetic factors

Short stature, low muscle mass, low nephron number

Longer survival of cardiovascular disease patients

Environmental factors

High salt intake

Obesity, metabolic syndrome

Drugs: non-steroidal anti-inflammatory drugs, antibiotics, contrast media

Social factors

Luxury acceptance for dialysis

phrology societies including that of Australia and New Zealand. The AFCKDI 2008 was held in Kuala Lumpur, Malaysia, along with the 11th Asian Pacific Congress of Nephrology on May 4, 2008. There were heated debates on the validity of the eGFR equation in Asia, specific considerations of etiology and risk factors of ESRD in Asia, and the international consideration and integration of the CKD initiative in Asia. There was still no consensus on the equation of eGFR for the Asian population, and several issues remain to be solved. There are several similarities and differences in the epidemiology of CKD in Asian countries. It was accepted that the strategy for CKD prevention, inter-relationship between developed and developing countries, and also among developing countries are important. The AFCKDI 2009 will be in Kaohsiung, Taiwan, on April 18-19, 2009. The strengths of this Forum are that it is becoming the Forum to get Asian colleagues together to discuss openly and exchange information. Future collaborative studies could be performed to compare the CKD incidence and prevalence in Asian countries using the Asian eGFR formula or adjusted formula. Hematuria is common in Asian populations, therefore more studies are needed concerning its significance on CKD progression.

\section{Perspectives}

Late referral to nephrologists is the case when dialysis is needed within 1 year after referral. We reported that $60 \%$ of the DM dialysis patients had presented with proteinuria, hypertension, or CVD at the time of DM diagnosis [18]. Automatic reporting of eGFR may alert other clinical disciplines to detect unrecognized CKD in their patients, which might otherwise develop to ESRD. Such a system might encourage physicians in non-nephrology services and increase the rate of referrals to nephrologists. Implementation of an eGFR reporting system should be associated with comprehensive education of practitioners. FROM-J is a good start of such a strategy in Japan. Early treatments are effective for incipient DM nephropathy $[19,20]$, therefore ESRD could be prevented. The significance of late referral to nephrologists and the high prevalence of ESRD remains to be studied in Japan. Currently, there is no available evidence that an early identification program lowers the incidence/prevalence of treated ESRD in the world.

CKD is common in Japan and is expected to increase, particularly in the elderly population (table 1). Proteinuria and hypertension are common in patients with CVD, DM, obesity, and metabolic syndrome. Further studies are necessary to determine the benefits of proteinuria screening and automatic reporting of eGFR on the future incidence of ESRD. Also, research is needed to determine (1) the natural course on CKD progression and (2) treatment options for CVD prevention associated with CKD.

Japan is making a concerted effort to intensify the national study of CKD and its repercussions. It is expected that over the next decade, information gathered through a number of initiatives may shed light on the nature of CKD in Japan and define national strategies.

References

1 Levey AS, Atkins R, Coresh J, et al: Chronic kidney disease as a global public health problem: approaches and initiatives - a position statement from Kidney Disease Improving Global Outcomes. Kidney Int 2007;72:247259.

2 Sarnak MJ, Levey AS, Schoolwerth AC, et al: Kidney disease as a risk factor for development of cardiovascular disease. A statement from the American Heart Association Councils on Kidney in Cardiovascular Disease, High Blood Pressure Research, Clinical Cardiology, and Epidemiology and Prevention. Circulation 2003;108:2154-2169.

- 3 Go AS, Chertow GM, Fan D, et al: Chronic kidney disease and the risks of death, cardiovascular events, and hospitalization. N Engl J Med 2004;351:1296-1305.

4 Nakai S, Wada A, Kitaoka T, et al: An overview of regular dialysis treatment in Japan (as of 31 December 2004). Ther Apher Dial 2006; 10:476-497.

5 Iseki K: Chronic kidney disease in Japan. Int Med 2008;47:681-689. 
6 Goodkin DA, Young EW, Kurokawa K, et al: Mortality among hemodialysis patients in Europe, Japan, and the United States: casemix effects. Am J Kidney Dis 2004;44(suppl 2):S16-S21.

$\checkmark 7$ Imai E, Yamagata K, Iseki K, et al: Kidney disease screening program in Japan, outcome and perspectives. Clin J Am Soc Nephrol 2007;2:1360-1366.

$\checkmark 8$ Imai E, Horio M, Yamagata K, et al: GFR decline rate in Japanese general population: a longitudinal 10 year follow-up study. Hypertens Res 2008;31:433-442.

$\checkmark 9$ Ninomiya T, Kiyohara Y: Chronic kidney disease and other diseases. 1. Cardiovascular diseases (in Japanese). Nippon Naika Gakkai Zasshi 2007;96:887-893.

10 Ninomiya T, Kiyohara Y, Kubo M, et al: Metabolic syndrome and CKD in a general Japanese population: the Hisayama Study. Am J Kidney Dis 2006;48:383-391.
1 Tozawa M, Iseki C, Tokashiki K, et al: Metabolic syndrome and risk of developing chronic kidney disease in Japanese adults. Hypertens Res 2007;30:937-943.

12 Yamagata K, Ishida K, Sairenchi T, et al: Risk factors for chronic kidney disease in a community-based population: a 10-year followup study. Kidney Int 2007;71:159-166.

$>13$ Iseki K: The Okinawa screening program. J Am Soc Nephrol 2003;14(suppl 2):S127S130.

14 Iseki K: Screening for renal disease - what can be learned from Okinawa experience? Nephrol Dial Transplant 2006;21:839-843.

15 Wen CP, Cheng TYD, Tsai MK, et al: Allcause mortality attributable to chronic kidney disease: a prospective cohort study based on 462,293 adults in Taiwan. Lancet 2008; 371:2173-2182.

16 Iseki K, Kinjo K, Iseki C, Takishita S: Relationship between predicted creatinine clearance and proteinuria and the risk of developing ESRD in Okinawa, Japan. Am J Kidney Dis 2004;44:806-814.
17 Imai E, Matsuo S, Makino H, et al: Chronic kidney disease Japanese Cohort (CKD-JAC) study: design and methods. Hypertens Res 2008;31:1101-1108.

18 Sunagawa H, Iseki K, Nishime K, et al: Epidemiologic analysis of diabetic patients on chronic dialysis. Nephron 1996;74:361-366.

19 Makino H, Haneda M, Babazono T, et al: Prevention of transition from incipient to overt nephropathy with telmisartan in patients with type 2 diabetes. Diabetes Care 2007;30:1577-1578.

20 Araki S, Haneda M, Koya D, et al: Reduction in microalbuminuria as an integrated indicator for renal and cardiovascular risk reduction in patients with type 2 diabetes. Diabetes 2007;56:1727-1730.

\title{
Editorial Comment
}

\author{
M. El Nahas, Sheffield
}

The minireview by Iseki highlights issues relating to CKD in Japan. Over the last 25 years, Japan has led the way in CKD screening strategies with data emanating from Okinawa identifying a number of communitybased risk factors. This editorial examines some of the known data and reviews new initiatives. It becomes apparent that, as elsewhere in the world, Japan may have a high prevalence of CKD; unfortunately, as with many reports, such a prevalence includes many with an eGFR between 50 and $59 \mathrm{ml} / \mathrm{min} / 1.73 \mathrm{~m}^{2}$ who may move in and out of CKD stage 3 based on serum creatinine estimation variability and/or may simply have age-related low GFR with little risk of progression. This is commented upon by the fact that progression seldom takes place in the absence of hypertension, diabetes or proteinuria. Iseki also introduces the reader to a number of Japanese and Asian CKD initiatives aimed at improving CKD awareness and planning strategies. It is encouraging to know that issues relating to CKD and its impact on global healthcare are prompting a number of national and regional initiatives. These are welcome in view of the paucity of solid data underlying a number of dogmatic statements relating to $\mathrm{CKD}$, its prevalence and complications. 